\title{
Combined Long-Period Fiber Grating and Microcavity In-Line Mach-Zehnder Interferometer for Refractive Index Measurements with Limited Cross-Sensitivity ${ }^{\dagger}$
}

\author{
Monika Janik ${ }^{1,2, *(\mathbb{D})}$, Marcin Koba ${ }^{1,3}{ }^{\oplus}$, Krystian Król ${ }^{1}$, Predrag Mikulic ${ }^{4}$, Wojtek J. Bock ${ }^{4}$ \\ and Mateusz Śmietana ${ }^{1}$ (D) \\ 1 Institute of Microelectronics and Optoelectronics, Warsaw University of Technology, Koszykowa 75, \\ 00-662 Warszawa, Poland; mkoba@elka.pw.edu.pl (M.K.); K.Krol@imio.pw.edu.pl (K.K.); \\ M.Smietana@elka.pw.edu.pl (M.Ś.) \\ 2 Department of Metrology and Optoelectronics, Faculty of Electronics, Telecommunications and Informatics, \\ Gdansk University of Technology, Narutowicza 11/12, 80-233 Gdansk, Poland \\ 3 The National Institute of Telecommunications, Szachowa 1, 04-894 Warszawa, Poland \\ 4 Centre de Recherche en Photonique, Université du Québec en Outaouais, 101 Rue St Jean Bosco Gatineau, \\ Gatineau, QC J8X 3X7, Canada; predrag.mikulic@uqo.ca (P.M.); wojtek.bock@uqo.ca (W.J.B.) \\ * Correspondence: monika.janik@pg.edu.pl or monika.janik@pw.edu.pl \\ + This paper is an extended version of Monika Janik; Marcin Koba; Predrag Mikulic; Wojtek J. Bock; \\ Mateusz Śmietana, Combined long-period grating and micro-cavity in-line Mach-Zehnder interferometer for \\ refractive index sensing, 25th International Conference on Optical Fiber Sensors, Jeju, Korea, 23 April 2017.
}

Received: 15 March 2020; Accepted: 23 April 2020; Published: 24 April 2020

\begin{abstract}
This work discusses sensing properties of a long-period grating (LPG) and microcavity in-line Mach-Zehnder interferometer ( $\mu \mathrm{IMZI})$ when both are induced in the same single-mode optical fiber. LPGs were either etched or nanocoated with aluminum oxide $\left(\mathrm{Al}_{2} \mathrm{O}_{3}\right)$ to increase its refractive index (RI) sensitivity up to $\approx 2000$ and $9000 \mathrm{~nm} / \mathrm{RIU}$, respectively. The $\mu$ IMZI was machined using a femtosecond laser as a cylindrical cavity $(\mathrm{d}=60 \mu \mathrm{m})$ in the center of the LPG. In transmission measurements for various RI in the cavity and around the LPG we observed two effects coming from the two independently working sensors. This dual operation had no significant impact on either of the devices in terms of their functional properties, especially in a lower RI range. Moreover, due to the properties of combined sensors two major effects can be distinguished-sensitivity to the RI of the volume and sensitivity to the RI at the surface. Considering also the negligible temperature sensitivity of the $\mu \mathrm{IMZI}$, it makes the combination of LPG and $\mu$ IMZI sensors a promising approach to limit cross-sensitivity or tackle simultaneous measurements of multiple effects with high efficiency and reliability.
\end{abstract}

Keywords: Mach-Zehnder interferometer (MZI); long-period grating (LPG); optical fiber sensors; refractive index sensing; femtosecond laser micromachining; thin films; multiparameter sensing; cross-sensitivity

\section{Introduction}

For many chemical and biological applications, precise monitoring of the refractive index (RI) of a liquid analyte is of great importance [1]. However, the highly accurate RI measurements depend on many factors such as temperature $(\mathrm{T})$ cross-sensitivity, especially for on-site applications, or surface sensitivity during the detection of small molecules as DNA targets, which can be easily disturbed by the $\mathrm{T}$ and the RI fluctuations, giving false results. In recent years, various fiber optical platforms incorporating multiple sensor elements have been used for multi-parameter sensing, especially for 
simultaneous measurements of the RI and the T. These include a reflection-mode long-period grating (LPG) with an intrinsic Fabry-Perot interferometer (FPI) [2], an FPI integrated with a fiber Bragg grating (FBG) [3], a dual LPG [4], a core-offset Mach-Zehnder interferometer (MZI) combined with FBG [5], or an FBG with microcavity in-line MZI ( $\mu$ IMZI) [6]. A review on this topic was presented by S. Pevec and D. Donlagic [7]. Each of these sensors has its limitations, e.g., the FBG- $\mu$ IMZI offers very high RI sensitivity, compact size, and precise sensing element location, but during simultaneous RI and $T$ measurements, the wavelength shift obtained for the interference minimum of the $\mu$ IMZI is approximately two orders of magnitude greater than the one for the resonant wavelength of the FBG. Thus, simultaneous tracing of the two shifts corresponding to RI and T was difficult and substantially limits practical applications of this platform. To overcome such issues, in this work we propose a sensor based on two the most RI sensitive optical fiber devices, namely the LPG and the $\mu$ IMZI. Both sensing concepts have been known and explored for decades for a great variety of sensing applications $[8,9]$.

The LPG is a periodic modulation of the RI along the length of the core in a single-mode optical fiber, which allows for coupling between the fundamental core mode and a series of cladding modes $[10,11]$. The coupling results in the appearance of resonances in the LPG transmission spectrum, where each of the resonances corresponds to the coupling of a certain cladding mode. The sensitivity of the LPG is typically defined as a spectral shift of the resonance wavelength with a measurand. Moreover, enhancing the LPG's RI sensitivity has been also explored and reported. It has been improved through tuning its working point [12] and coating the grating with a high-RI nano-overlay [13]. Thus, currently, LPGs offer sensitivity exceeding 10,000 nm/RIU for measurements in proximity to the sensor's surface [14]. However, because of its high-temperature sensitivity, there is always a measurement error resulting from the cross-sensitivity [15]. To counter this problem, a control over T of the investigated liquid during the measurements is typically applied [16].

According to the MZI concept, the light beam splits into two parts propagating along two different paths, which interfere with one another after a certain distance [17]. When guiding conditions in one of the paths are disturbed, the effect can be identified by tracking changes in the interference pattern. The $\mu$ IMZI structures presented in this paper are fabricated in a standard telecom optical fiber using femtosecond laser ablation [17], where the light propagates in the fiber core and splits at the cavity wall into reference (core) and sensing (cavity) beams, each with different optical lengths. When the beams reach the opposite wall of the cavity, they interfere like the two beams in an MZI. Thanks to the size reaching tens of micrometers and application of fs laser micromachining, the device is compact, highly reproducible, and well-suited for applications where as low as $\mathrm{pL}$ volumes are investigated. Moreover, it may offer negligible T sensitivity and very high RI sensitivity reaching over 20,000 nm/RIU [17,18].

In this paper, we discuss an ability for combining the two sensing devices, namely LPG and $\mu I M Z I$, into a single compact sensing system. Based on the sensing scheme proposed here, multiple measurands can be calculated from a spectrum acquired using a single spectrum analyzer. The two optical fiber sensors are both capable of high-sensitivity RI measurements with one major difference: the LPG is best suited for RI measurements close to the fiber's surface while the $\mu$ IMZI has been applied in bulk RI measurements, where the surface effect can be ignored [19]. When, e.g., biological molecules from a liquid sample under analysis attach to the surface of a functionalized sensor, the observed output signal corresponds to the growth of a biological layer. This sensing phenomenon and the effect of surface RI sensitivity often form the basis for label-free sensing using LPGs [20,21]. Although it is proven that for LPG both types of RI sensitivities can be optimized to some extent, it applies only for simple RI measurements using liquids. Since there is no difference between liquid properties at the surface and away from the LPG, the same measurement results are correct for RI in volume. However, when only thin biofilm at the LPG's surface needs to be identified the measurements gain complexity. In this case, the result of the measurement is a superposition of RI changes at the surface and in the volume, and at this point, only these at the surface are of interest. That is why for this exact application, we propose to discriminate these two kinds of sensitivity using LPG response associated mainly with the surface RI sensitivity $\left(\mathrm{S}_{\mathrm{S}}\right)$, and the $\mu \mathrm{IMZI}$ response with the volume RI 
sensitivity $\left(\mathrm{S}_{\mathrm{v}}\right)$. At washing steps, only the response from LPG will change due to the appearance of thin biofilm, while part of the spectrum corresponding to $\mu$ IMZI will stay unchanged. Thus, the sensing configuration allows for clear identification of the changes at the surface. Considering also the negligible $T$ sensitivity of the $\mu \mathrm{IMZI}$, the combination of LPG and $\mu$ IMZI sensors makes a promising approach to tackle simultaneous measurements of multiple effects with high efficiency and reliability.

\section{Materials and Methods}

\subsection{Fabrication of $L P G$}

A set of LPGs was fabricated in hydrogen-loaded germanium-doped Corning SMF-28 single-mode optical fibers by UV irradiation of a 5-cm long fiber section with a $\mathrm{KrF}$ excimer laser [22]. The fibers were exposed through a chromium amplitude mask with a period of $226.8 \mu \mathrm{m}$. The period of the grating has been optimized to reach the RI sensitivity around $2000 \mathrm{~nm} / \mathrm{RIU}$ within the RI range between 1.333 and $1.350 \mathrm{RIU}$, and to obtain dispersion turning point (DTP) between 1500 and $1600 \mathrm{~nm}$ [23]. After the UV-writing, the LPGs were annealed at $150{ }^{\circ} \mathrm{C}$ for $3 \mathrm{~h}$ to release the hydrogen and to stabilize the properties of the gratings. Next, a set of LPGs was chemically etched in hydrofluoric (HF) acid up to the DTP taking place at an external RI =1.3330 RIU (Figure 1A1) [20]. Considering the etching rate of $10 \% \mathrm{HF}$ acid [2] and the etching time, it is estimated that changes in the cladding thickness can reach up to $5 \mu \mathrm{m}$. Thus, the final diameter of the cladding decreased to ca. $115 \mu \mathrm{m}$. The estimated values have been confirmed by simulations [20,24]. The other set of LPGs was coated with aluminum oxide $\left(\mathrm{Al}_{2} \mathrm{O}_{3}\right)$ nano-overlay according to the procedure reported in [14] (Figure 1A2). This nanocoating with a high-RI material allowed us to optimize RI sensitivity by obtaining both mode transition (MT) and DTP effects [12].

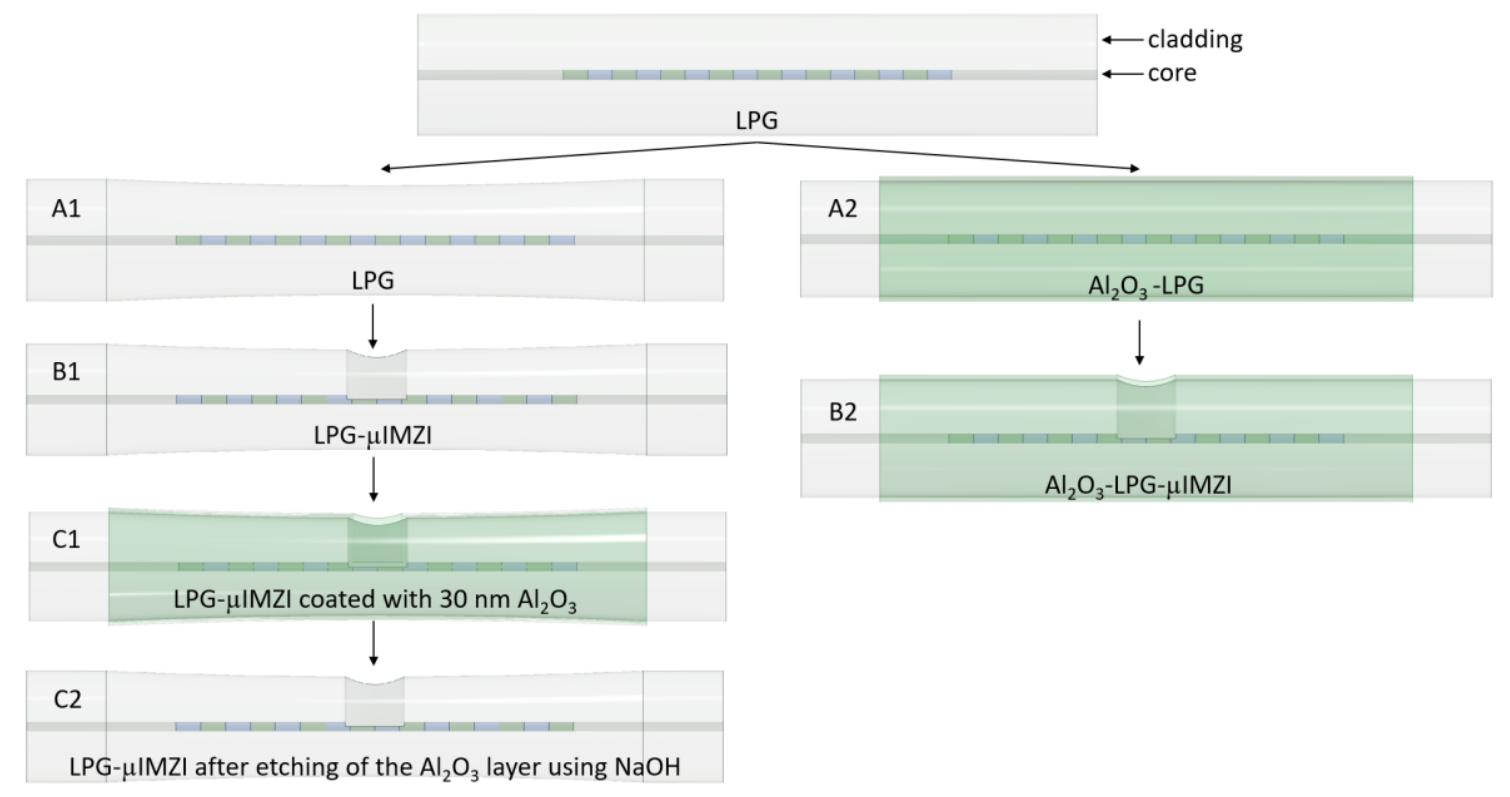

Figure 1. Schematic visualization of the sample preparation, where (A1) represents a long-period grating (LPG) etched with hydrofluoric (HF) acid; (A2) — an LPG nanocoated with aluminum oxide $\left(\mathrm{Al}_{2} \mathrm{O}_{3}\right) ;($ B1) - a combination of the microcavity in-line Mach-Zehnder interferometer $(\mu \mathrm{IMZI})$ and etched LPG; (B2)—a combination of the $\mu$ IMZI and nanocoated LPG; (C1)—a LPG- $\mu$ IMZI coated with $30 \mathrm{~nm} \mathrm{Al}{ }_{2} \mathrm{O}_{3}$ layer as a simulation of a biofilm formation; (C2) - a LPG- $\mu$ IMZI after etching of the $\mathrm{Al}_{2} \mathrm{O}_{3}$ layer with sodium hydroxide. Features are not to scale.

\subsection{Fabrication of $\mu I M Z I$}

The cylindrical cavity ( $60 \mu \mathrm{m}$ diameter) was micromachined in the middle of the LPG through the cladding and core reaching half of the fiber $(60 \mu \mathrm{m}$ depth) (Figure 1B1,B2). The structure has a circular 
cross-section, flat bottom, while its walls, with good approximation, are perpendicular to the fiber's long axis [25]. The cavity diameter of $60 \mu \mathrm{m}$ was chosen due to mechanical constraints and for ease of liquid application. Micromachining was performed using a Solstice Ti:Sapphire fs laser operating at $\lambda=795 \mathrm{~nm}$. The fiber was irradiated by $82 \mathrm{fs}$ pulses. To make the microcavity, the laser beam was directed into a suitably designed micromachining setup based on the Newport $\mu$ Fab system. The system was equipped with a $20 \times$ lens, with $N A=0.30$. During the fabrication, the fiber's transmission spectrum was monitored with an NKT Photonics SuperK COMPACT supercontinuum white light source and a Yokogawa AQ6370C optical spectrum analyzer. The fabrication process was controlled with software developed in-house.

\section{3. $L P G$ and $L P G-\mu I M Z I$ Analysis}

First, the spectral response of the LPG was investigated in the wavelength range from 1100 to $1700 \mathrm{~nm}$ using the light source and spectrum analyzer described in Section 2.2. The RI sensitivity was identified by immersing the LPGs in water/glycerin mixture with RI ranging from 1.3330 to 1.4000 RIU. The RIs of the liquids were measured using a VEE GEE PDX-95 digital refractometer with a resolution of $10^{-4}$ RIU. Before the first immersion in the liquids, the $\mathrm{Al}_{2} \mathrm{O}_{3}$-nanocoated LPG- $\mu$ IMZI underwent $30 \mathrm{~s}$ long oxygen plasma processing, which increased the wettability of the film's surface and made filling the cavity with the liquids possible [17]. The RI of liquids was verified just after the transmission measurements by taking them directly from the vicinity of the fiber sample. Between immersions, the LPG was rinsed with deionized water. T and strain were kept constant during all the RI measurements. The T measurements were performed while the structures were immersed in water. We used a home-made aluminum flow-cell where the bottom part was T-controlled in the range $10-45^{\circ} \mathrm{C}$. A reference $\mu$ IMZI structure also underwent the same testing procedure. Figure 2 depicts the schematic representation of the measurement setup. The measurements were conducted on three etched and three nanocoated LPGs with fabricated microcavities. Each measurement was conducted in five repetitions to check the stability of the sensor and measurement setup. The following results present the representative spectra averaged from five consecutive measurements of two chosen sensors, namely LPG- $\mu$ IMZI and $\mathrm{Al}_{2} \mathrm{O}_{3}$-LPG- $\mu$ IMZI.

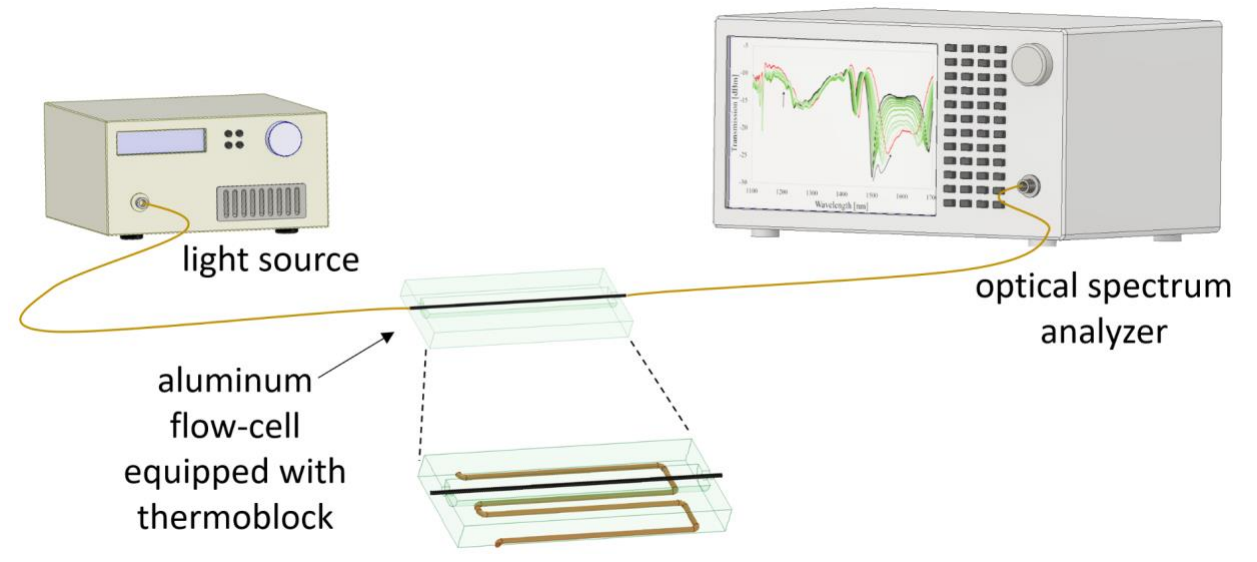

Figure 2. Schematic representation of the measurement setup.

\subsection{Monitoring of RI Changes at the Surface with LPG- $\mu$ MZI.}

The $30 \mathrm{~nm}$ in thickness $\mathrm{Al}_{2} \mathrm{O}_{3}$ nano-overlay was deposited on LPG- $\mu$ MZI following the procedure described in [10] (Figure 1C1). Next, the $\mathrm{Al}_{2} \mathrm{O}_{3}$ thickness was reduced on LPG and the microcavity by the sensor immersion in a sodium hydroxide $(\mathrm{NaOH})$ of known concentration $(10 \mathrm{mM}$ and $1 \mathrm{M})$ at a fixed temperature $\left(\mathrm{T}=20^{\circ} \mathrm{C}\right)$ for a specified time [26], followed by extensive washing with deionized water (Figure 1C2). The optical transmission spectrum of the $\mu \mathrm{IMZI}$ was investigated during the 
etching and compared when the sensor was immersed in deionized water. Before the first $\mathrm{NaOH}$ etching, the cavity underwent 30-s long oxygen plasma processing, as mentioned in Section 2.1.

\section{Results and Discussion}

\subsection{RI Sensing with LPG or $\mu$ MZI Structure}

The spectral responses of the LPG and $\mathrm{Al}_{2} \mathrm{O}_{3}$-nanocoated LPG $\left(\mathrm{Al}_{2} \mathrm{O}_{3}\right.$-LPG) to RI before $\mu$ IMZI micromachining are shown in Figure $3 \mathrm{~A}, \mathrm{~B}$, respectively. An increase of the spectral distance between the resonances is observed when the RI increases, which is very characteristic for this type of sensing structure working at the DTP of higher-order cladding modes [14]. The RI sensitivity for each resonance is close to 2000 and $9000 \mathrm{~nm} / \mathrm{RIU}$ for LPG and $\mathrm{Al}_{2} \mathrm{O}_{3}$-LPG, respectively. Due to the higher RI sensitivity of the $\mathrm{Al}_{2} \mathrm{O}_{3}-\mathrm{LPG}$, the resonances shift significantly more with RI.
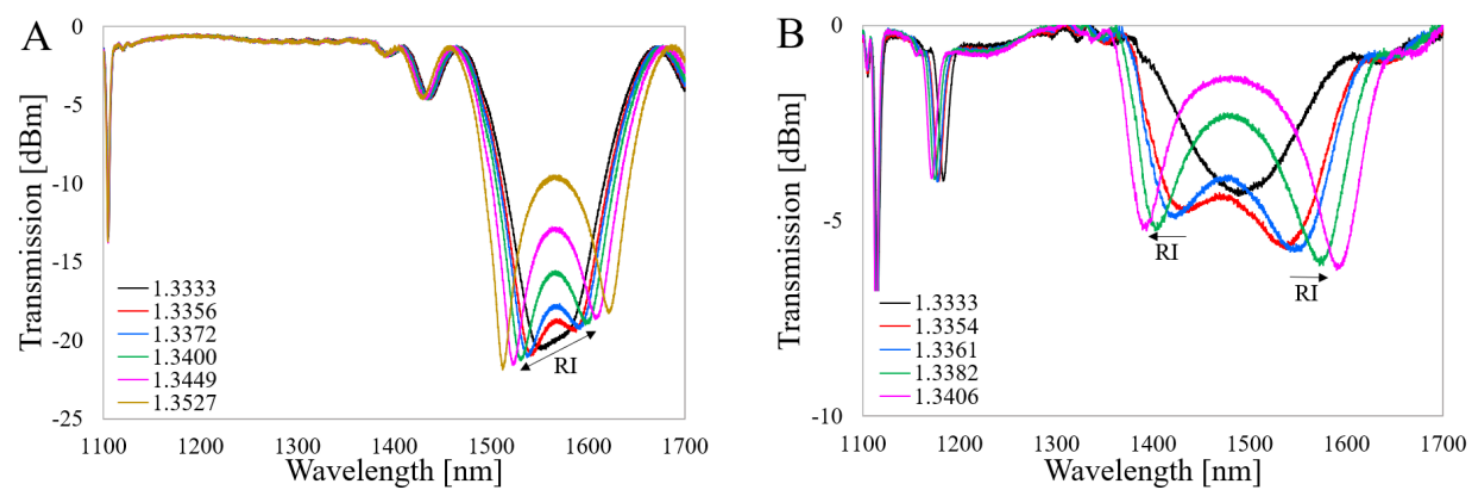

Figure 3. Spectral response of the investigated structures (A) LPG and (B) $\mathrm{Al}_{2} \mathrm{O}_{3}$-LPG to changes in refractive index (RI). The arrows indicate a spectral shift with external RI.

In Figure 4 , in turn, the spectral response of a reference $\mu$ IMZI is shown. This structure was micromachined separately for comparison. The microcavity has the same diameter $d=60 \mu \mathrm{m}$ as the $\mu$ IMZIs fabricated later in the LPGs. In general, we observe here a shift of the transmission minima towards shorter wavelengths with increasing RI values in the cavity. The substantiation of the spectrum evolution was presented in our previous work [27]. It is worth mentioning that due to the micromachined interferometric structure, the overall transmission dropped by ca. $7-10 \mathrm{~dB}$. This effect is induced by the formation of the $\mu$ IMZI cavity, which makes direct interaction between the fiber core and the external medium possible. The RI sensitivity of the presented $\mu$ IMZI reaches $15,000 \mathrm{~nm} / \mathrm{RIU}$.

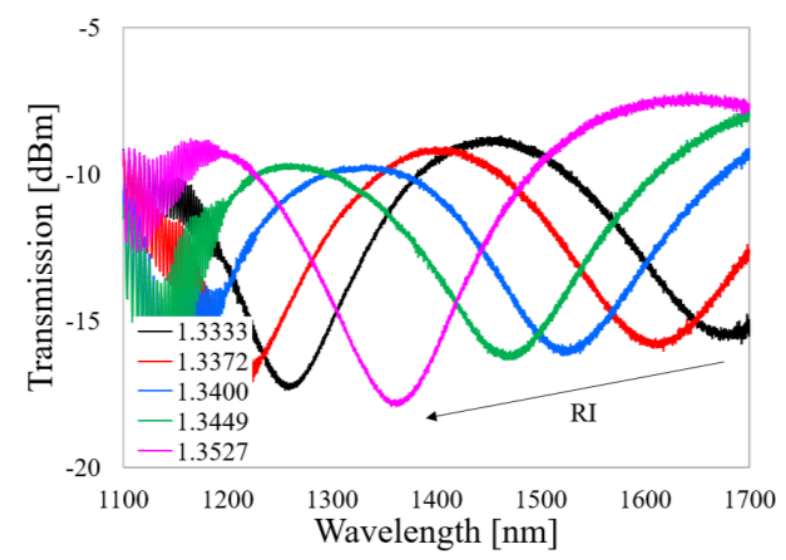

Figure 4. Spectral response of the reference $\mu \mathrm{IMZI}$ with cavity diameter $d=60 \mu \mathrm{m}$. An arrow indicates the shift of the minimum with RI. 


\subsection{RI Sensing with LPG- $\mu$ MZI Structures}

Figure 5A,B shows the spectral response to RI in its different ranges for the LPG after $\mu$ IMZI micromachining, i.e., with the microcavity in the middle of the LPG.
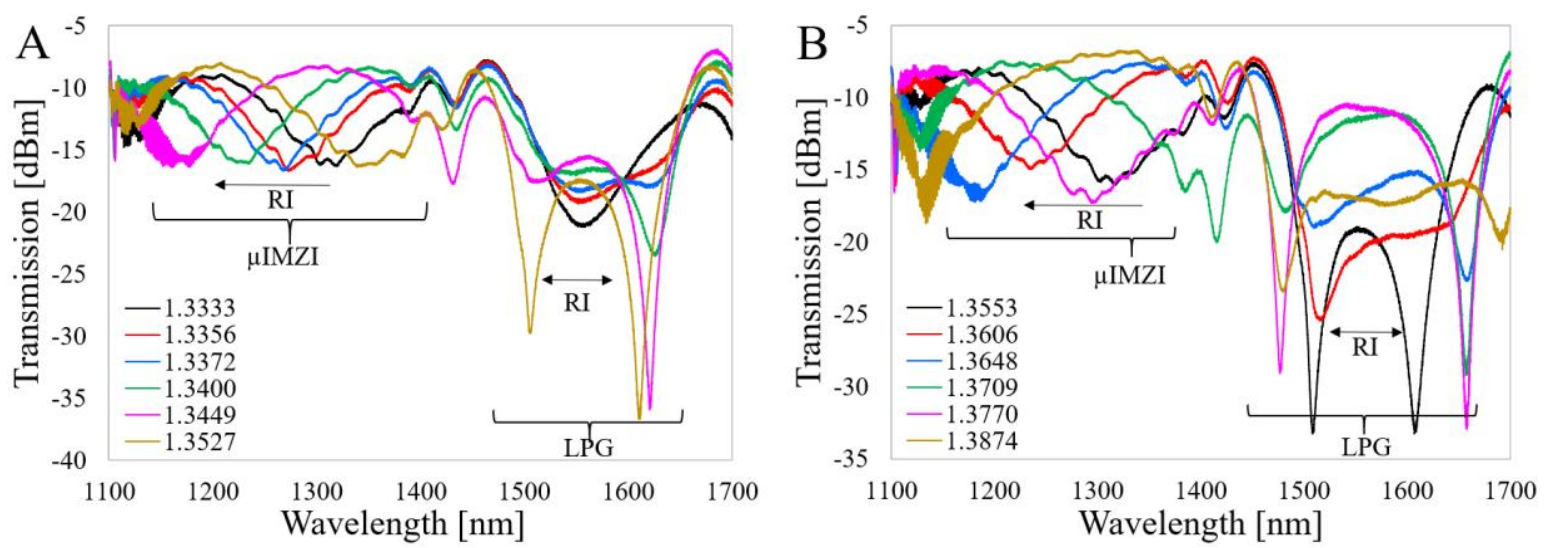

Figure 5. Spectral response of the LPG- $\mu$ IMZI structure to RI in ranges: (A) from 1.3333 to 1.3527 RIU and (B) from 1.3553 to 1.3874 RIU. The effects corresponding to the LPG and $\mu$ IMZI are marked in the figures.

It is interesting that the LPG still ordinarily responds to the RI despite a significant discontinuity in the fiber cladding. Given that the LPG's working principle relies on the cladding modes, it is perhaps counter-intuitive that the microcavity had no direct effect on the LPG response. The micromachining process led to an increase in the overall insertion loss (transmission dropped by ca. 10-14 dB). The presence of the cavity also manifests itself in the oscillatory character of the spectrum, which in this case is caused by scattered waves at the cavity interferences. Finally, two specific ranges in the spectrum are evident, above and below $1400 \mathrm{~nm}$. The higher part resembles the spectrum of the LPG before micromachining (Figure $3 \mathrm{~A}$ ), while the lower one is dominated by the response characteristic to the $\mu$ IMZI (Figure 4). Based on the curves shown in Figures 5 and 6, we can state that the LPG- $\mu$ IMZI response is a composite of the LPG and $\mu$ IMZI spectra. Figure 5 shows that with an increase of the $\mathrm{RI}$, the spectral response of the combined device is similar to the responses of the LPG and $\mu \mathrm{IMZI}$ working independently, which is more evident for smaller values of RI. Specifically, we observe an increase of the resonance wavelength separation in the range of 1400-1700 nm, which corresponds to the profile of the LPG. There is also a blue shift of the minima in the 1200-1400 nm range which, in turn, typically corresponds to responses of the $\mu$ IMZI (Figure 5A). For higher values of RI, the second minima, characteristic for the $\mu \mathrm{IMZI}$, appear at the higher wavelengths and mingle with the response induced by the LPG. This causes distortions in the spectrum and makes the two effects interfere with one another (Figure 5B). The dominant regions for each effect are still distinguishable, but very much disturbed, especially in the case of the minima characteristic for the LPGs. The interfering effects in some RI ranges are critical and this fact needs to be taken into consideration while designing the device.

Next, the RI-induced spectral evolution for the $\mathrm{Al}_{2} \mathrm{O}_{3}$-LPG with microcavity was investigated (Figure 6).

As in the case of the LPG, the overall transmitted power after micromachining is significantly reduced. The transmission dropped by ca. $7-11 \mathrm{~dB}$. Thanks to the higher sensitivity of the $\mathrm{Al}_{2} \mathrm{O}_{3}-\mathrm{LPG}$, the effect is even more noticeable at higher RI values. On the other hand, the more sensitive the LPG structure is, the more distortion and undulations appear in the spectrum after the micromachining. Although it was always possible to determine one of the LPG's minima, as well as the minimum for the $\mu \mathrm{IMZI}$, the obtained spectrum was not that smooth and well pronounced as in the case of the sensors working separately. 
A few possible causes for the origins of these distortions are, e.g., exciting additional modes, or introducing a phase shift to the already existing one. It is also worth reminding that both the sensing structures were made in a standard single-mode fiber where the cutoff wavelength is $\lambda=1260 \mathrm{~nm}$. Below this value, the fiber works in a multimode regime and the high-frequency oscillations in the spectrum are observed mainly in the short wavelength region. As shown in Figure 6A, the minimum at around $1200 \mathrm{~nm}$ corresponding to the $\mu \mathrm{IMZI}$ diminishes with RI, i.e., moves towards the shorter wavelengths and cannot be further observed due to the cut-off wavelength of the fiber. Nevertheless, the second minimum can be seen above RI of 1.3416 RIU at about 1600-1700 nm (Figure 6B).
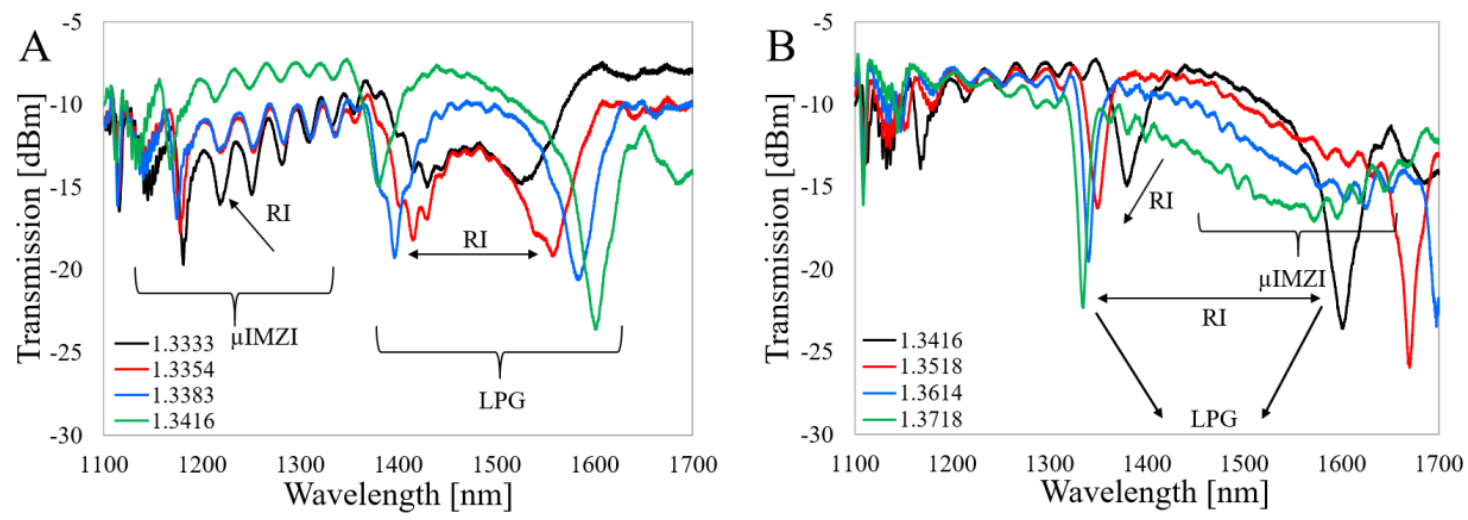

Figure 6. Spectral responses of the $\mathrm{Al}_{2} \mathrm{O}_{3}$-LPG- $\mu \mathrm{IMZI}$ structure to $\mathrm{RI}$ in its range (A) from 1.3333 to 1.3416 RIU and (B) from 1.3416 to 1.3718 RIU. The effects corresponding to the $\mathrm{Al}_{2} \mathrm{O}_{3}$ - $\mathrm{LPG}$ and $\mu$ IMZI are marked.

It can be concluded that the results obtained for the two types of LPGs with $\mu$ IMZIs differ only quantitatively. The similarities are explained by the fact that both have the same effect and the same general principle of operation, while the differences have several reasons. The spectra shown in Figure 6 indicate that the minimum corresponding to the $\mu \mathrm{IMZI}$ is in the short wavelength region. The second minimum, in contrast to the LPG, emerges for relatively lower RI values. Moreover, due to the considerably higher sensitivity of the $\mathrm{Al}_{2} \mathrm{O}_{3}$-LPG, its resonances shift significantly more with the RI and the $\mu$ IMZI effect is more pronounced and less distorted than for higher RI in case of the LPG. It might be expected that a RI range exists for which the distortion makes discrimination of the effects difficult. However, in the investigated cases during the whole experiment, i.e., for the entire RI range, it was always possible to determine one of the LPG's minima, as well as the minimum of the $\mu \mathrm{IMZI}$. It is important to note that in this case, i.e., $\mathrm{Al}_{2} \mathrm{O}_{3}$ - $\mathrm{LPG}$, the exact definition of the location of the minima requires additional signal processing, e.g., curve fitting or pattern recognition. Furthermore, along with some additional signal processing, the full-width half minimum (FWHM) parameter could also be improved, especially for the $\mu$ IMZI part of the spectrum. Even though its reduction requires additional fine-tuning of the sensor, such as reactive ion etching as reported in our previous work [28]. In this discussion, we must acknowledge that also the placement of the cavity might influence the transmission spectrum of the device. In the case of both structures, despite the significant discontinuity in the fiber cladding, which sustains the cladding modes, the LPG-related effect remains valid and the cavity does not affect the output of the sensor significantly.

From the above discussion, we can conclude that the two effects, the first stemming from the LPG and the second from the $\mu \mathrm{IMZI}$, seem to be independent. Furthermore, we have seen that in lower RI ranges they do not affect each other and can be well separated. This separation of effects may be used to reduce the cross-sensitivity of the proposed device. Since the lower RI range of operation is essential in many applications, including bio-sensing, the combined structure could well serve for taking measurements where two different RI sensitivity effects are expected. Experiments such as those reported here have been carried out previously for both platforms separately [14,19], but never for a 
combination of the platforms. The prospect of being able to simultaneously detect and distinguish the earlier mentioned different sensitivities (surface and volume) effects using a two-in-one platform serves as a continuous incentive for ongoing studies and motivates future research.

\subsection{Monitoring of RI at the Surface with the LPG- $\mu M Z I$}

In the previous sections, we discussed the high $S_{V}$ of the sensor. However, the primary motive behind the combination of the LPG and $\mu$ IMZI was rather a determination of the difference between $S_{V}$ and $S_{S}$. Based on the working principles of each platform it can be stated that the LPG is best suited for detecting surface RI changes, while the $\mu$ IMZI excels in volume RI measurements, where the surface effect can be ignored to some extent. To prove our hypothesis and simulate the biological film formation, we deposited a high-RI thin overlay of $\mathrm{Al}_{2} \mathrm{O}_{3}$ using the ALD method on the entire LPG- $\mu$ IMZI. ALD provided a highly controllable and uniform $30 \mathrm{~nm}$ layer all over the LPG- $\mu$ IMZI sensor. The $\mathrm{Al}_{2} \mathrm{O}_{3}$ can be dissolved in both highly concentrated acids and alkalis, which enables studying the response of the sensor to different thicknesses of the thin film [26]. In Figure 7 the influence of the $\mathrm{Al}_{2} \mathrm{O}_{3}$ etching process on the spectral response of the LPG- $\mu$ IMZI is shown. The arrows indicate the change of the spectral response with the progression of the etching process.

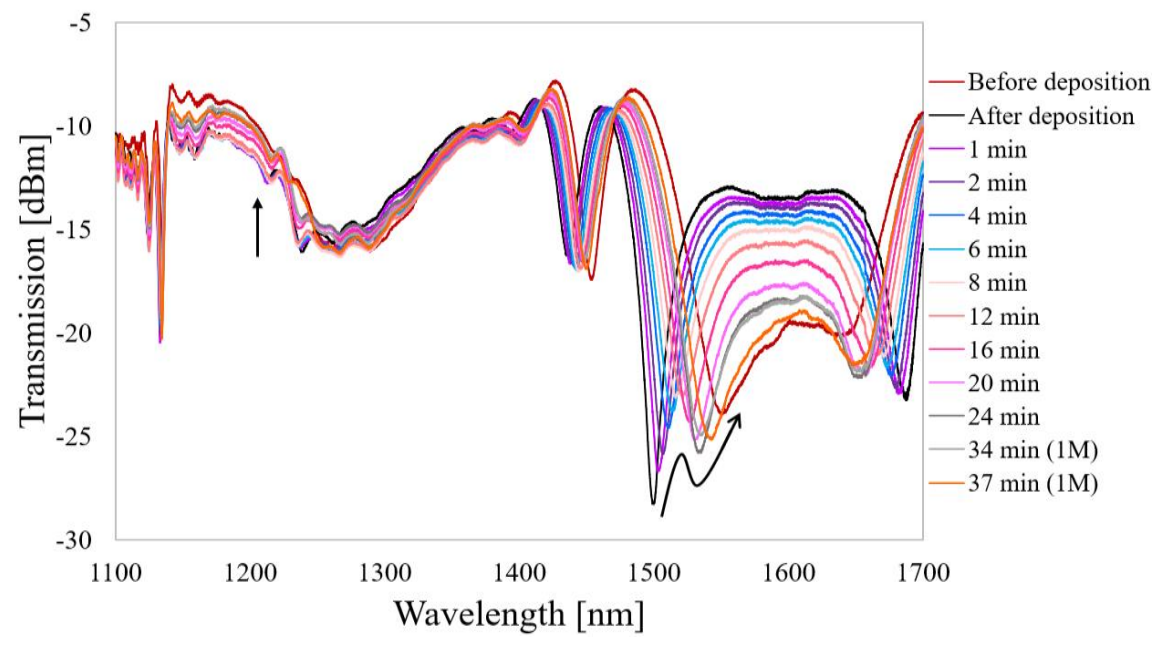

Figure 7. The response of LPG- $\mu$ IMZI to $\mathrm{Al}_{2} \mathrm{O}_{3}$ film etching recorded for measurements in water. The plot shows the evolution of the transmission spectrum during the process of the response before the deposition. Due to the low effectiveness of the etching process, the last two etching rounds were performed in $1 \mathrm{M} \mathrm{NaOH}$.

The most apparent change concerns the LPG part of the spectrum. LPGs are highly sensitive towards external RI including even the thinnest overlay formed on their surface. Any change of the thickness of the nanocoating manifests itself as a shift of the resonance wavelength, and it was observed after the deposition process. Both resonances shifted away from each other for about $50 \mathrm{~nm}$ from the initial stage. Moreover, the LPG operates in the proximity of the DTP. Thus, the deposition process splits both resonances and makes them apparent and defined. Slow etching in $10 \mathrm{mM} \mathrm{NaOH}$ induced the shift of the spectrum (indicated by black arrows) and with time the resonances were getting close to each other, and finally, they got back to the initial working point. In contrast to the response from the LPG, the $\mu$ IMZI part of the spectrum only slightly reacted by the deposited $\mathrm{Al}_{2} \mathrm{O}_{3}$ overlay. We can observe the changes mainly in terms of the transmission amplitude. The properties of the deposited layer-its RI and thickness—did not change the working conditions of the $\mu$ IMZI. In conclusion, if we are targeting detection of surface changes (ca. tens of nm overlays), it will be too small to affect the volume sensitivity of the $\mu$ IMZI part of the sensor and in effect, it will stay unrecognizable to the $\mu I M Z I$, or it will induce changes just in terms of transmission amplitude. However, combined with surface-sensitive LPG, the sensor can stand as a perfect tool to measure the growth of an overlay and, 
for example, simultaneous fluctuations of the external RI. This, in turn, could find the application in the detection of biological targets such as DNA aptamers, which are a key target in medical diagnostic tests.

Figure 8 presents a schematic comparison of three cases when the use of combined LPG- $\mu$ IMZI would allow to deliver enriched information about the analyzed thin film, including biofilm, and the surrounding liquid. Figure $8 \mathrm{~A}$ shows a situation when both the LPG and $\mu$ IMZI are influenced by a high-RI liquid $\left(n_{1}\right)$. Figure $8 B$, in turn, presents a case where a very thin layer $\left(h_{2}\right)$, such as a biological film is deposited, and the external RI is lower than in the case shown in Figure $8 \mathrm{~A}\left(\mathrm{n}_{2}<\mathrm{n}_{1}\right)$. The last example (Figure 8 C) concerns a thicker layer $\left(h_{3}>h_{2}\right)$ and the lowest $\mathrm{RI}\left(\mathrm{n}_{3}<\mathrm{n}_{2}\right)$. For the LPG, the measurement will be a superposition of effect coming from RI, the thickness of the nanolayer, and to some extent RI of the surrounding medium. Thus, on the LPG part of the spectrum for specific values of $\mathrm{n}_{1}, \mathrm{n}_{2}$, and $\mathrm{n}_{3}$, as well as $\mathrm{h}_{2}$ and $\mathrm{h}_{3}$ there will be no or slight difference between thin and thicker film surrounded by higher and lower external RI. Only the contribution from $\mu$ IMZI would allow to discriminate the cases and identify changes in RI and, in consequence, changes in film growth, too. During the biological experiments, the discrimination of such differences is crucial, e.g., the thickness of the film may correspond to the concentration of biological targets, while external RI indicates proper removal of excess of the unbound targets. Thus, the proposed sensing combination enables clear identification of the changes at the surface and in its proximity, as well as further interpretation of the results.

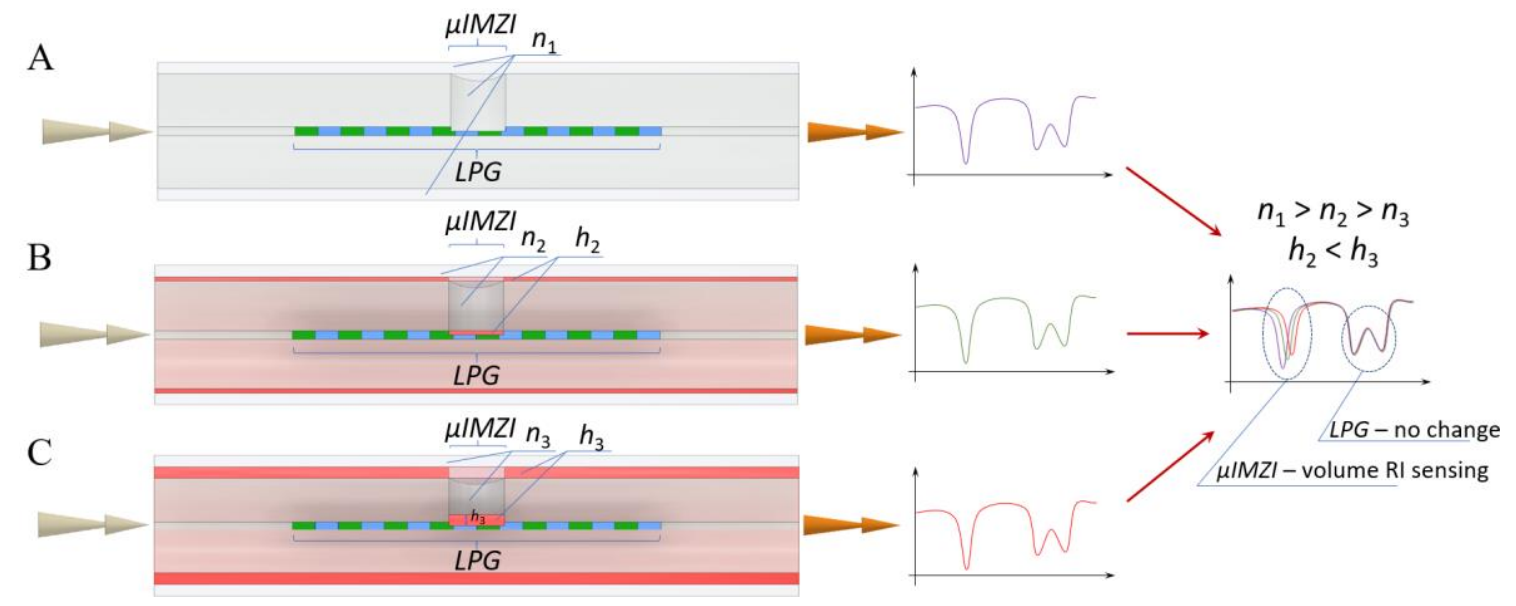

Figure 8. A schematic showing LPG- $\mu$ IMZI influenced by (A) high external RI, (B) thin film and lower external RI, and (C) thick film and the lowest external RI. For this specific combination of external RIs $\left(\mathrm{n}_{1}, \mathrm{n}_{2}\right.$, and $\left.\mathrm{n}_{3}\right)$ and film thicknesses, only the response of $\mu$ IMZI would allow discriminating the cases.

\subsection{Temperature Sensitivity of LPG- $\mu I M Z I$}

In the preceding Sections 3.2 and 3.3, it was shown that the combined structures work independently and do not affect each other's operation in any significant way. This can be even more interesting when one realizes that in addition to a slight reaction to the thin overlay deposition, in contrast to the single LPG, the $\mu$ IMZI is almost T-insensitive [18]. To demonstrate the T-insensitive RI sensing with the $\mu I M Z I$, the reference $\mu$ IMZI was placed in a T-controlled cell and immersed in water. The transmission spectrum was monitored while the $\mathrm{T}$ was gradually increased from 10 to $45^{\circ} \mathrm{C}$ with a $5{ }^{\circ} \mathrm{C}$ step. The spectra obtained during these measurements show that with an increase of $\mathrm{T}$, the minimum shifted towards longer wavelengths (Figure 9).

The RI of the fiber core, as well as diameter of the microcavity, are treated as constants over the range of the $\mathrm{T}$ applied during the described experiment $\left(10-45^{\circ} \mathrm{C}\right)$ considering the thermo-optic coefficient $\left(6.3 \times 10^{-6} /{ }^{\circ} \mathrm{C}\right)$ and thermal expansion coefficient $\left(0.55 \times 10^{-6} /{ }^{\circ} \mathrm{C}\right)$ of the fiber materials $[29,30]$. The obtained T sensitivity of the $\mu \mathrm{IMZI}$ in water is $1.2 \mathrm{~nm} /{ }^{\circ} \mathrm{C}$ at ca. $1250 \mathrm{~nm}$ and is induced mainly by RI sensitivity of the structure which is $\approx 15,000 \mathrm{~nm} / \mathrm{RIU}$. Since the thermo-optic coefficient of water 
reaches $-1 \times 10^{-4} \mathrm{RIU} /{ }^{\circ} \mathrm{C}$ and is two orders of magnitude higher than that of fused silica $\left(6.3 \times 10^{-6} /{ }^{\circ} \mathrm{C}\right)$, we can conclude that the observed shift of the minimum is induced almost exclusively by the change in the RI of water caused by $\mathrm{T}$ variation.

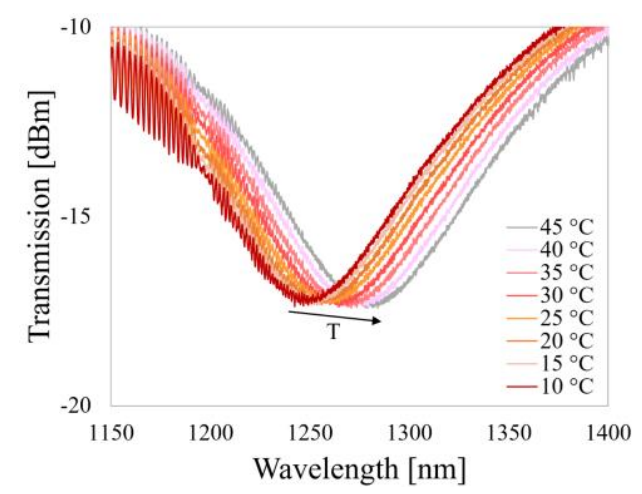

Figure 9. Transmission spectra of $\mu \mathrm{IMZI}$ at different temperatures (T) of water in the cavity.

In Figure 10 evolution of $\mathrm{Al}_{2} \mathrm{O}_{3}-\mathrm{LPG}-\mu \mathrm{IMZI}$ transmission spectra with $\mathrm{T}$ is presented. Here, we see that with the increase of $\mathrm{T}$, the spectral distance between the resonances of the LPG decreases, while the part dominated by the microcavity response barely changes. Assuming that the two effects are independent and that the change of the spectrum caused by the $\mu \mathrm{IMZI}$ is relatively small when compared to that caused by the LPG, we can infer that the sensor is suited for highly accurate RI measurements in cases where the $\mathrm{T}$ of the investigated medium varies.

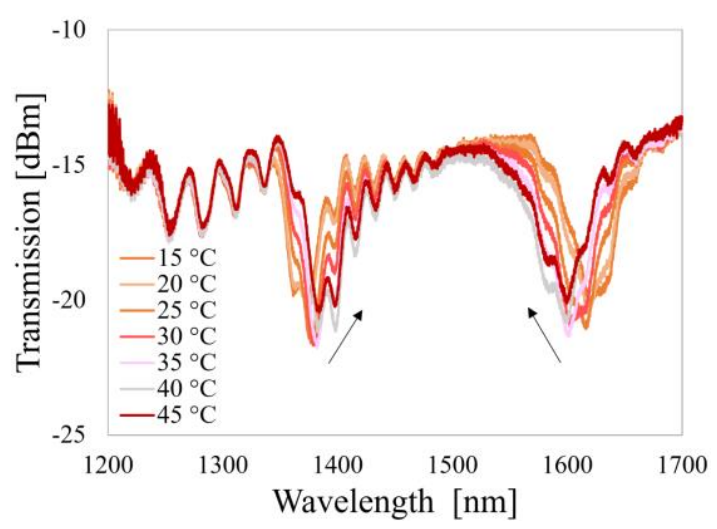

Figure 10. Spectral responses of the $\mathrm{Al}_{2} \mathrm{O}_{3}-\mathrm{LPG}-\mu \mathrm{IMZI}$ structure in water to variations of $\mathrm{T}$.

Multiparameter sensing is often unavoidable to acquire desired information on the performance of a chosen system. Thus, in recent years many configurations have been presented, especially for $\mathrm{T}$ and RI sensing. However, just two other sensors are incorporating a grating and in-fiber cavities. The first sensor was created by combining the micromachined cavities in-between two FBGs [31]. The second was made by a combination of FBG with the $\mu$ IMZI [6]. In both cases, the FBGs provided very high sensitivity. However, because of the combination, as indicated in the introduction, differences in the values of the traced wavelength shifts limit the practical application of the sensors. The design and manufacturing method of the first-mentioned sensor also highly bounds its performance. Two microcavities made in one fiber significantly weakened the sensing structure. Besides, the reproducibility of the sensor is questionable due to the Excimer laser processing combined with highly uncontrollable hydrofluoric acid etching. Regardless of the combination, both sensors provided the information about $\mathrm{T}$ and RI. Therefore, they cannot be compared in terms of surface and volume sensitivities. This is the first sensor to date which has been considered for such application. 


\section{Conclusions}

This work presents a device containing LPG and $\mu$ IMZI in the same single-mode optical fiber. Two LPGs, tuned towards high RI sensitivity by fiber cladding etching $(2000 \mathrm{~nm} / \mathrm{RIU})$ and $\mathrm{Al}_{2} \mathrm{O}_{3}$ nanocoating deposition ( $9000 \mathrm{~nm} / \mathrm{RIU})$, were later modified by fabrication of cylindrically shaped cavities. These microcavities with diameters of $d=60 \mu \mathrm{m}$ were micromachined in the middle of each of the 5-cm long LPGs. During the experiments, it was found that in both cases, they responded as two independently working sensors, one associated with the grating and the other with the microcavity. At some values of the RI, an overlapping took place. The problem of distortions or overlapping can be overcome by additional signal processing, e.g., curve fitting or pattern recognition, however, it needs further studies as it may limit the operation range of the sensor. Nevertheless, this effect has no significant impact on the independent operation of the sensors in the specified lower RI ranges, which are required by many applications, e.g., bio-sensing. Although neither of the platforms improved the other one, they perfectly complemented each other and they both had exceptional RI sensitivity (LPG at surface and $\mu \mathrm{IMZI}$ in volume). Thus, this combination allowed to obtain more in-depth information about the measurand through multi-parameter sensing. Especially small bio-targets, such as DNA aptamers, should be considered where the LPG part would be responsible for specific label-free recognition and the $\mu \mathrm{IMZI}$ 's signal would be responsible for tracing the RI and its fluctuations caused by, e.g., temperature distortions.

Author Contributions: Conceptualization, M.J., M.K. and M.Ś.; Software, M.K.; Resources, K.K., P.M.; W.J.B.; Methodology, M.K., M.Ś.; Validation, M.J., M.K., and M.Ś.; Formal Analysis, M.J., M.K., and M.Ś.; Investigation, M.J. and M.K.; Data Curation, M.J.; Writing_Original Draft Preparation, M.J., M.K. and M.Ś.; Writing—Review and Editing, M.J., M.K., M.Ś.; Visualization M.K. and M.J.; Supervision, W.J.B. and M.Ś.; Funding Acquisition, M.Ś. and W.J.B.; Project administration: M.Ś. and W.J.B. All authors have read and agreed to the published version of the manuscript.

Funding: This work was supported in Poland by the National Science Center (NCN) grant No. 2018/29/B/ST7/02552 and National Centre for Research and Development (NCBiR) as a part of Techmatstrateg grant No. 347324/12/NCBR/2017.

Conflicts of Interest: The authors declare no conflict of interest.

\section{References}

1. Wang, X.D.; Wolfbeis, O.S. Fiber-optic chemical sensors and biosensors (2015-2019). Anal. Chem. 2020, 92, 397-430. [CrossRef] [PubMed]

2. Kim, D.W.; Shen, F.; Chen, X.; Wang, A. Simultaneous measurement of refractive index and temperature based on a reflection-mode long-period grating and an intrinsic Fabry-Perot interferometer sensor. Opt. Lett. 2005, 30, 3000. [CrossRef] [PubMed]

3. Chen, L.X.; Huang, X.G.; Li, J.Y.; Zhong, Z.B. Simultaneous measurement of refractive index and temperature by integrating an external Fabry-Perot cavity with a fiber Bragg grating. Rev. Sci. Instrum. 2012, 83, 053113. [CrossRef] [PubMed]

4. Yan, J.; Zhang, A.P.; Shao, L.; Ding, J.; He, S.; Member, S. Simultaneous measurement of refractive index and temperature by using dual long-period gratings with an etching process. Sensors (Peterborough, NH) 2007, 7, 1360-1361. [CrossRef]

5. Yao, Q.; Meng, H.; Wang, W.; Xue, H.; Xiong, R.; Huang, B.; Tan, C.; Huang, X. Simultaneous measurement of refractive index and temperature based on a core-offset Mach-Zehnder interferometer combined with a fiber Bragg grating. Sens. Actuators A Phys. 2014, 209, 73-77. [CrossRef]

6. Liao, C.R.; Wang, Y.; Wang, D.N.; Yang, M.W. Fiber in-line Mach-Zehnder interferometer embedded in FBG for simultaneous refractive index and temperature measurement. IEEE Photonics Technol. Lett. 2010, 22, 1686-1688. [CrossRef]

7. Pevec, S.; Donlagić, D. Multiparameter fiber-optic sensors: A review. Opt. Eng. 2019, 58, 1. [CrossRef]

8. James, S.W.; Tatam, R.P. Optical fibre long-period grating sensors: Characteristics and application. Meas. Sci. Technol. 2003, 14. [CrossRef] 
9. Zhao, Y.; Zhao, H.; Lv, R.; Zhao, J. Review of optical fiber Mach-Zehnder interferometers with micro-cavity fabricated by femtosecond laser and sensing applications. Opt. Lasers Eng. 2019, 117, 7-20. [CrossRef]

10. Vengsarkar, A.M.; Lemaire, P.J.; Judkins, J.B.; Bhatia, V.; Erdogan, T.; Sipe, J.E. Long-period fiber gratings as band-rejection filters. J. Light. Technol. 1996, 14, 58-64. [CrossRef]

11. Shu, X.; Zhang, L.; Bennion, I. Sensitivity characteristics of long-period fiber gratings. J. Light. Technol. 2002, 20, 255-266. [CrossRef]

12. Śmietana, M.; Koba, M.; Mikulic, P.; Bock, W.J. Combined plasma-based fiber etching and diamond-like carbon nanooverlay deposition for enhancing sensitivity of long-period gratings. J. Light. Technol. 2016, 34, 4615-4619. [CrossRef]

13. Śmietana, M.; Myśliwiec, M.; Mikulic, P.; Witkowski, B.S.; Bock, W.J. Capability for fine tuning of the refractive index sensing properties of long-period gratings by atomic layer deposited $\mathrm{Al} 2 \mathrm{O} 3$ overlays. Sensors 2013, 13, 16372-16383. [CrossRef]

14. Śmietana, M.; Koba, M.; Mikulic, P.; Bock, W.J. Towards refractive index sensitivity of long-period gratings at level of tens of $\mu \mathrm{m}$ per refractive index unit: Fiber cladding etching and nano-coating deposition. Opt. Express 2016, 24, 11897. [CrossRef]

15. Grochowski, J.; Myśliwiec, M.; Mikulic, P.; Bock, W.J.; Smietana, M. Temperature cross-sensitivity for highly refractive index sensitive nanocoated long-period gratings. Acta Phys. Pol. A 2013, 124, 421-424. [CrossRef]

16. Janczuk-Richter, M.; Dominik, M.; Koba, M.; Mikulic, P.; Bock, W.J.; MacKowski, S.; Jonsson-Niedziolka, M.; Niedziolka-Jonsson, J.; Smietana, M. Water-induced fused silica glass surface alterations monitored using long-period fiber gratings. J. Light. Technol. 2019, 37, 4542-4548. [CrossRef]

17. Janik, M.; Myśliwiec, A.K.; Koba, M.; Celebańska, A.; Bock, W.J.; Śmietana, M. Sensitivity pattern of femtosecond laser mach-zehnder interferometers, as applied to small-scale refractive index sensing. IEEE Sens. J. 2017, 17, 3316-3322. [CrossRef]

18. Hu, T.Y.; Wang, D.N. Optical fiber in-line Mach-Zehnder interferometer based on dual internal mirrors formed by a hollow sphere pair. Opt. Lett. 2013, 38, 3036-3039. [CrossRef]

19. Śmietana, M.; Janik, M.; Koba, M.; Bock, W.J. Transition between bulk and surface refractive index sensitivity of micro-cavity in-line Mach-Zehnder interferometer induced by thin film deposition. Opt. Express 2017, 25, 26118-26123. [CrossRef]

20. Brzozowska, E.; Śmietana, M.; Koba, M.; Górska, S.; Pawlik, K.; Gamian, A.; Bock, W.J. Recognition of bacterial lipopolysaccharide using bacteriophage-adhesin-coated long-period gratings. Biosens. Bioelectron. 2015, 67, 93-99. [CrossRef]

21. Piestrzyńska, M.; Dominik, M.; Kosiel, K.; Janczuk-Richter, M.; Szot-Karpińska, K.; Brzozowska, E.; Shao, L.; Niedziółka-Jonsson, J.; Bock, W.J.; Śmietana, M. Ultrasensitive tantalum oxide nano-coated long-period gratings for detection of various biological targets. Biosens. Bioelectron. 2019, 133, 8-15. [CrossRef] [PubMed]

22. Smietana, M.; Bock, W.J.; Mikulic, P.; Ng, A.; Chinnappan, R.; Zourob, M. Detection of bacteria using bacteriophages as recognition elements immobilized on long-period fiber gratings. Opt. Express 2011, 19, 7971-7978. [CrossRef] [PubMed]

23. Śmietana, M.; Koba, M.; Mikulic, P.; Bock, W.J. Measurements of reactive ion etching process effect using long-period fiber gratings. Opt. Express 2014, 22, 5986-5994. [CrossRef]

24. Śmietana, M.; Dominik, M.; Mikulic, P.; Bock, W.J. Temperature and refractive index sensing with Al2O3-nanocoated long-period gratings working at dispersion turning point. Opt. Laser Technol. 2018, 107, 268-273. [CrossRef]

25. Janik, M.; Member, S.; Eftimov, T.; Koba, M.; Smietana, M.; Bock, W.J.; Member, L. Tailoring properties of microcavity in-line mach-zehnder interferometer by the microcavity enlargement using femtosecond laser. J. Light. Technol. 2019, 37, 4501-4506. [CrossRef]

26. Śmietana, M.; Mikulic, P.; Bock, W.J. Nano-coated long-period gratings for detection of sub-nanometric changes in thin-film thickness. Sens. Actuators A Phys. 2018, 270, 79-83. [CrossRef]

27. Janik, M.; Koba, M.; Celebańska, A.; Bock, W.J.; Śmietana, M. Live E. coli bacteria label-free sensing using a microcavity in-line Mach-Zehnder interferometer. Sci. Rep. 2018, 8. [CrossRef]

28. Janik, M.; Koba, M.; Celebańska, A.; Bock, W.J.; Śmietana, M. Sensing properties of micro-cavity in-line Mach-Zehnder interferometer enhanced by reactive ion etching. Opt. Laser Technol. 2018, 103, 260-266. [CrossRef] 
29. Ran, Z.L.; Rao, Y.J.; Liu, W.J.; Liao, X.; Chiang, K.S. Laser-micromachined Fabry-Perot optical fiber tip sensor for high-resolution temperature-independent measurement of refractive index. Opt. Express 2008, 16, 2252. [CrossRef]

30. Smietana, M.; Bock, W.J.; Mikulic, P. Temperature sensitivity of silicon nitride nanocoated long-period gratings working in various surrounding media. Meas. Sci. Technol. 2011, 22. [CrossRef]

31. Liu, Y.; Liu, X.; Ma, C.; Zhou, Y. Micro-structured optical fiber sensor for simultaneous measurement of temperature and refractive index. Opt. Fiber Technol. 2018, 41, 168-172. [CrossRef]

(C) 2020 by the authors. Licensee MDPI, Basel, Switzerland. This article is an open access article distributed under the terms and conditions of the Creative Commons Attribution (CC BY) license (http://creativecommons.org/licenses/by/4.0/). 